\title{
Cartografía de cañones: Alto Tajo y cañón del Júcar en la Manchuela. Problemas Metodológicos
}

María José González Amuchastegul* y Antonio Fernández Fernández ${ }^{\star \star}$

\section{INTRODUCCIÓN}

La representación cartográfica es uno de los aspectos de mayor importancia en los estudios geográficos. Sin embargo su tratamiento varía mucho de unos trabajos a otros, bien porque cada tema exige un tipo de cartografía determinada, o por las características específicas del espacio analizado. Así, refiriéndonos a los estudios geomorfológicos, la representación cartográfica entraña ciertas dificultades en áreas dominadas por altas plataformas subhorizontales, incididas por una red fluvial encajada que da lugar a profundas gargantas y angostos cañones, los cuales, albergan la mayor parte de la información geomorfológica del territorio.

Con este trabajo exponemos la metodología empleada para solucionar estos problemas en dos territorios alejados y con fuertes diferencias geológicas y estructurales: el Alto Tajo y el cañón del Júcar en La Manchuela. No obstante guardan ciertas afinidades morfológicas, al caracterizarse ambos por la existencia de amplias superficies surcadas por profundos cañones, así como, por la presencia de numerosos testigos morfológicos indicadores de la compleja evolución cuaternaria.

\section{EL ALTO TAJO-PARAMERAS DE MOLINA DE ARAGÓN Y EL CAÑÓN DEL JÚCAR EN LA MANCHUELA}

El territorio del Alto Tajo aquí analizado se inscribe en la rama castellana del Sistema Ibérico, en la provincia de Guadalajara (figura 1), dentro

* UPV. Departamento de Geografía.

* UNED. Departamento de Geografía 
del sector definido por los grandes ejes estructurales que orientados de NW a SE organizan la Cadena.

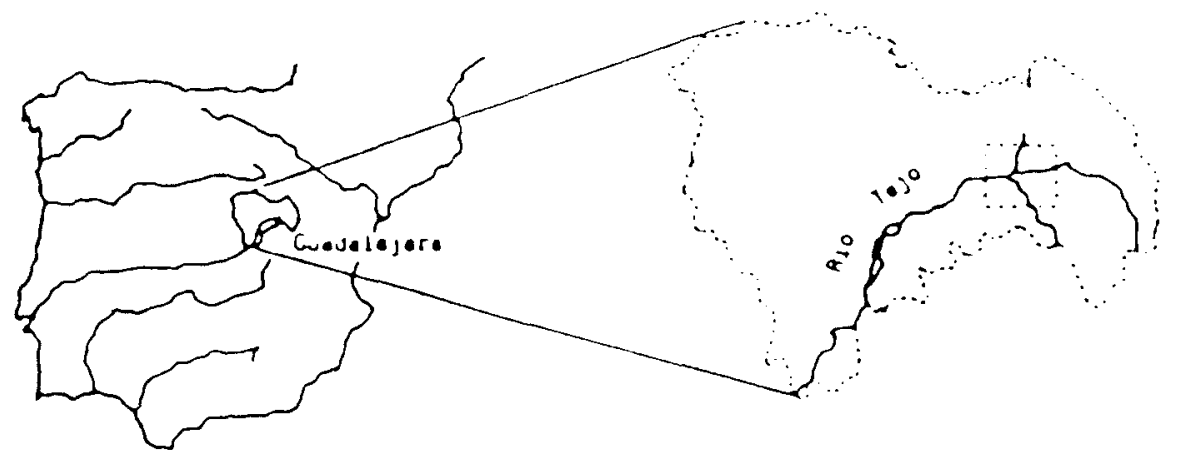

Figura 1. Localización del Alto Tajo.

En este área la red fluvial ha labrado un conjunto de angostos valles sobre los materiales mesozoicos, calizos y dolomíticos, adaptándose a un entramado tectónico de intersecciones direccionales alpinas: NW-SE en el caso de éstas y NE-SW para las guadarrámicas. Se trata de un relieve plegado en el que dominan las estructuras muy laxas, casi de disposición subhorizontal, como ocurre con el Cretácico superior determinando la aparición de relieves en forma de "mesa» cuya continuidad morfológica y tectónica se ve interrumpida bruscamente por el profundo valle del Tajo; éste ha aprovechado para labrar su valle, un conjunto de apretados pliegues de dirección SE-NW, de gran longitud y con flancos de fuerte buzamiento.

La suavidad de la morfología cimera se ha visto reforzada por el desarrollo de una extensa superficie de erosión, dando como resultado el claro predominio de las formas subhorizontales. En este ámbito, al que debe unirse la dominancia de las litologías calizas, el desarrollo e implantación de la red fluvial se ha visto muy dificultado; en efecto, la circulación superficial encauzada, a excepción del Tajo y de alguno de sus tributarios, es rara. Frente a ello se ha producido una intensa karstificación, con un predominio de la circulación subterránea y gran desarrollo de las formas endokársticas (simas, cuevas), frente a otro más moderado de las formas superficiales, entre las que destacan pequeñas dolinas, un poljé y otras depresiones cerradas, lejos de la espectacularidad que estas morfologías alcanzan en parajes vecinos de Cuenca y Teruel. 


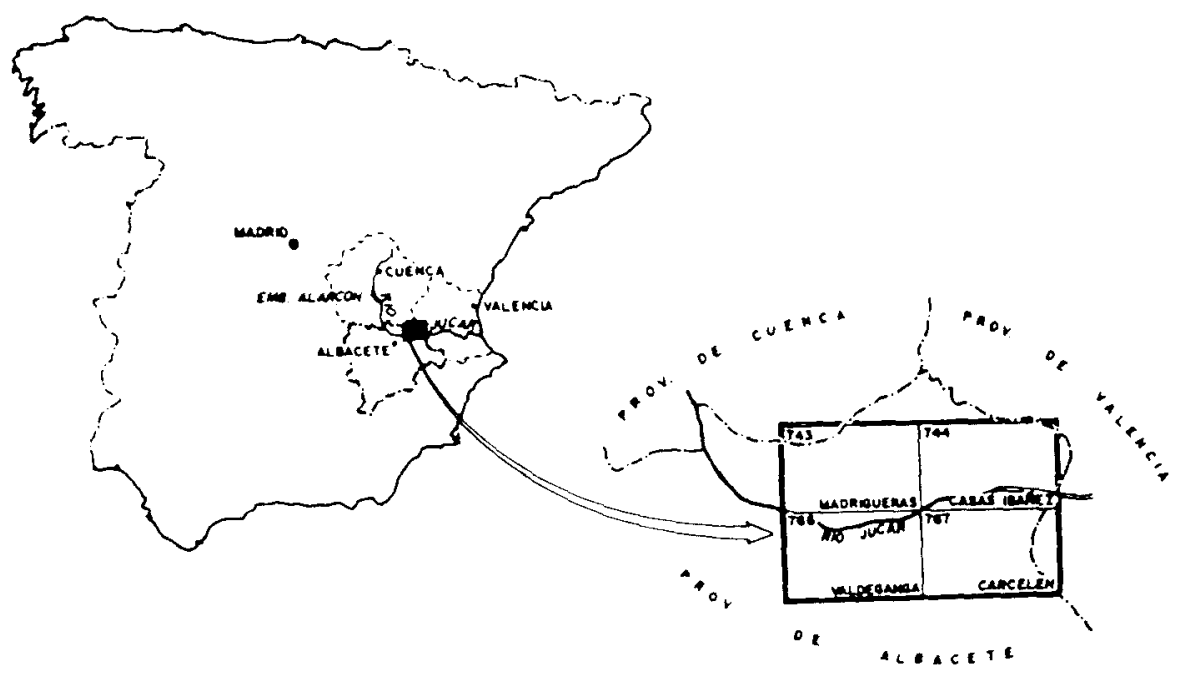

Figura 2. Localización de la Manchuela.

Dentro de la misma región de Castilla-La Mancha pero en sector nororiental de la provincia de Albacete se localiza la segunda área de estudio: el cañón del Júcar en la comarca de La Manchuela (figura 2).

Comparte con el Alto Tajo una morfología similar, es decir, una vasta superficie subhorizontal sobre la que se ha encajado una profunda hoz labrada por el río Júcar. Sin embargo, esta similitud morfológica encubre unas fuertes diferencias que se pueden resumir en los siguientes puntos.

* El principal conjunto litológico está constituido por potentes bancos de calizas lacustres de edad pliocena. Este roquedo apenas presenta procesos de karstificación, lo cual supone una importante diferencia respecto a la representación cartográfica del Alto Tajo.

* El conjunto carbonático de la fosa del Júcar presenta una diposición tabular, de tal forma que la dinámica fluvial no ha estado mediatizada por las direcciones de plegamiento como en el caso del Alto Tajo.

* La superficie culminante de La Manchuela no se corresponde con una superficie de erosión como en el caso de la Paramera de Molina de Aragón.

A pesar de estas diferencias estructurales la evolución cuaternaria de los río Tajo y Júcar han generado espectaculares cañones y han dejado diversas formaciones acumulativas, que actúan cómo testigos geomorfológicos de su evolución cuaternaria. 
En ambos casos, y a pesar de las diferencias señaladas, estos elementos han constituido claves esenciales para la interpretación evolutiva de los cañones, igualmente, han supuesto una serie de problemas cartográficos similares en los trabajos de investigación realizados en sendos territorios. (GONZÁLEZ AMUCHASTEGUI, M. ${ }^{a}$ J., 1993, y FERNÁNDEZ FERNÁNDEZ, A., 1996).

Problemas y soluciones en la elaboración de la cartografía del Alto Tajo y del Júcar

Como se ha indicado más arriba la elaboración de una cartografía adecuada está determinada en gran medida por las características físicas del área a representar. En los ámbitos analizados pueden diferenciarse en este sentido dos grandes elementos morfológicos. Por un lado, las amplias y abiertas superficies en las que tan sólo aparecen como elementos destacables algunos ejemplos y de escasa definición de morfologías exokársticas, especialmente en el Alto Tajo, y por otro la red fluvial que discurre profundamente encajada albergando en su interior las claves para el estudio geomorfológico de estos territorios.

La propia dinámica fluvial del Tajo y del Júcar, tanto en su aspecto quimico como detrítico, han generado una sucesión de depósitos y acumulaciones tobáceas, así como, numerosos niveles de acumulación fluvial, cuyo tamaño planteaba un importante problema a la hora de cartografiarlos.

Los edificios y acumulaciones tobáceas se corresponden a diferentes etapas evolutivas a lo largo del cuaternario. Su valioso significado paleoambiental y paleoclimático (GONZÁLEZ AMUCHASTEGUI, M. ${ }^{a} \mathrm{~J} .$, y GONZÁLEZ MARTÍN, 1989) (FERNÁNDEZ, A.; GARCÍA DEL CURA, M. a A.; GONZÁLEZ, J.A., y ORDÓÑEZ, S., 1996), han servido para establecer la correspondiente evolución cronológica y geomorfológica.

Respecto a los niveles de acumulación fluvial, han sido numerosos los retazos hallados a diferentes alturas a lo largo de los valles estudiados. En la mayor parte de los casos no presentan morfología de terrazas y constituyen pequeños afloramientos de escala métrica.

Por último la dinámica de vertientes ha dado lugar a numerosos depósitos de laderas que reflejan la sucesión de etapas de biostasia y rexistasia. 
Esta coexistencia de dos elementos morfológicos de características espaciales contrapuestas y niveles de información geomorfológica contrastada, plantea un primer gran problema en la elaboración cartográfica.

El primer aspecto a solucionar es la selección de una escala adecuada para la representación de estas unidades morfológicas; efectivamente, el empleo de 1/50.000 resulta idóneo para la representación de las altas plataformas culminantes, sin embargo, es claramente insuficiente para la representación de los cañones. Por el contrario el uso de una escala mayor, soluciona el problema de la representación de las gargantas, pero supone un resultado poco satisfactorio para la cartografía de las superficies culminantes, al no aportar mayor información y hacer que los mapas resultantes sean excesivamente grandes y de difícil manejo.

Este problema fue abordado de diferente forma en los dos territorios analizados.

En el caso del Alto Tajo se optó por usar diferentes escalas.

a) Se utilizó la escala 1/200.000 o inferiores para la representación de los aspectos generales del área (geología, conjuntos kársticos, localización de depósitos, red hidrográfica, etc.) (figura 3).

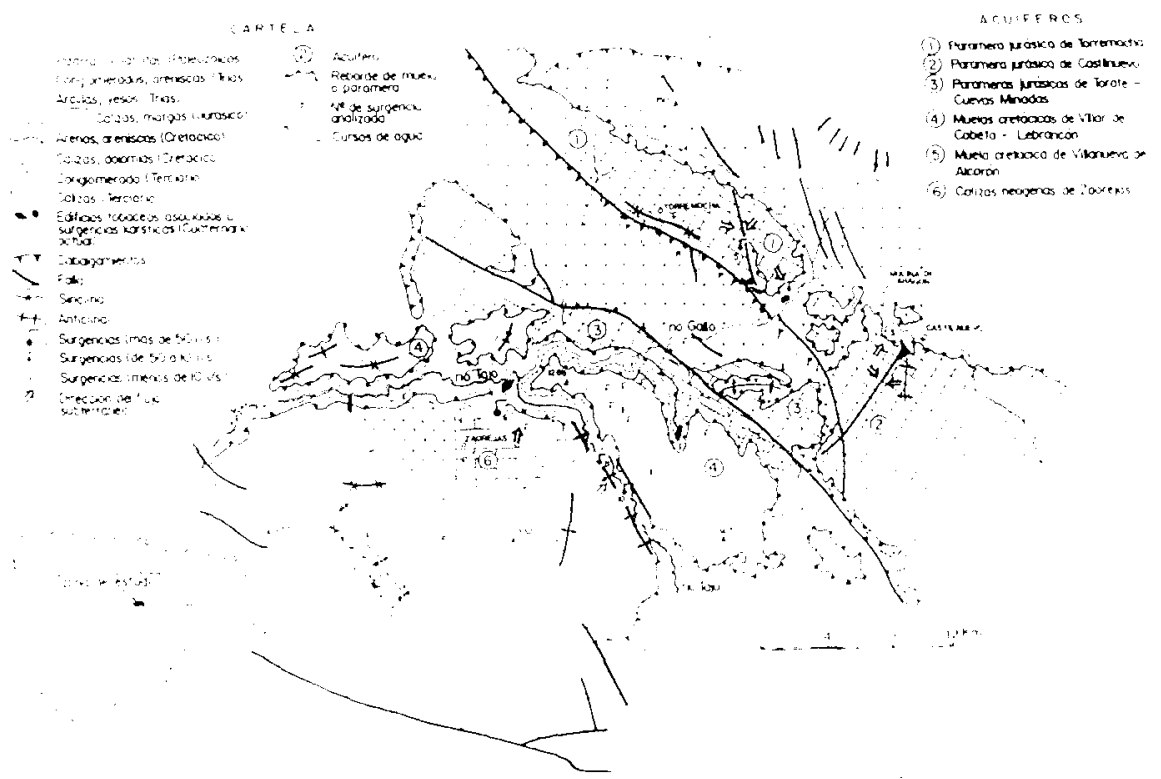

Figura 3. Localización de los principales acuiferos en el área de Molina de Aragón. 
b) La escala $1 / 50.000$ se utilizó para la cartografía de las grandes unidades en las que se dividió el territorio y en la que quedan planteados los principales problema geomorfológicos del Alto Tajo y Parameras de Molina. De este modo en un único mapa se obtiene una visión de conjunto de toda la comarca, siendo satisfactorio el nivel informativo aportado tanto por las plataformas altas que ya no recibirán un tratamiento más detallado, como por los profundos valles en los que quedan ya planteados los principales problemas que serán objeto de un análisis más pormenorizado en una cartografía a mayor escala.

c) Por último, la escala 1/25.000 sirvió para representar con suficiente detalle el interior de los profundos valles, plasmando ya la variada tipología y cronología de los depósitos fluviales y coluvionares.

En ocasiones, ha sido necesario recurrir a escalas más detalladas, sobre todo en los puntos en los que el cañón se hacía más estrecho, o bien, donde los depósitos presentaban una estructura más compleja, optándose por representaciones a escala 1/10.000.

En el caso de la hoz del Júcar debido a la menor extensión estudiada y a las características de la investigación, donde el objetivo principal es la propia hoz y el análisis de la superficie culminante constituye un objetivo secundario, se optó por utilizar una única escala $1 / 25.000$ y reducir al máximo la extensión de la superficie culminante cartografiada. Sin embargo, esta opción planteó problemas de realización , puesto que, el único mapa disponible era el de escala 1/50.000. Para solventar este problema se realizó una ampliación reprográfica, en la cual se localizaron los elementos geomorfológicos que, previamente, se habian ubicado en la fotografía aérea a escala $1 / 30.000$.

A pesar del detalle geomorfológico alcanzado a las escala 1/25.000 y $1 / 10.000$, había tramos de los valles en los que éstas se mostraban insuficientes para cartografiar la información geomorfológica. Este hecho obedece a la complejidad de ciertos edificios sedimentarios, de escasa entidad morfológica, pero de vital información paleoambiental, derivada de la superposición de acumulaciones tobáceas, aluviales y coluvionares, generadas bajo condiciones climáticas muy contrastadas.

La solución adoptada en ambos territorios fue la siguiente:

1. Establecimiento de un corte topográfico-geológico a gran escala (1/1000 o superior). En él se sitúan en sus posiciones geomorfológicas y altimétricas los cuerpos sedimentarios. 
2. Levantamiento de la columna estratigráfica de detalle de las diferentes acumulaciones, en la que se muestra la disposición de los lechos, la naturaleza y su estructura interna (figura 4).

Con el anterior método nos aseguramos una representación gráfica que complementa a los mapas geomorfológicos, los cuales, debido a la escala utilizada, no hubiesen permitido el nivel de detalle aportado por los gráficos (transectos-columnas estratigráficas). De lo anterior se deduce que la utilización de ambas técnicas se complementa y sólo es recomendable en aquellos casos en los que la complejidad del edificio, la minuciosidad de los análisis y la información paleoambiental que aportan, sean lo suficientemente importantes para justificar el trabajo gráfico y el nivel de información que conlleva.

Por último, como complemento a la cartografía geomorfológica,en ciertos tramos de los valles, se han realizado modelos polifásicos de la evolución cuaternaria del relieve (figura 5). El levantamiento de los bloquediagramas evolutivos presenta ciertas dificultades derivadas de la necesidad de abundantes testigos geomorfológicos en el campo, capaces de atestiguar las distintas etapas acaecidas, y que nos permitan establecer la secuencia evolutiva. La realidad muestra que los procesos erosivos dejan escasos afloramientos de calidad y fiabilidad geomorfológica suficiente, como para sustentar en ellos deducciones y afirmaciones paleoambientales precisas y fiables.

En el supuesto de la existencia de los testigos geomorfológicos suficientes, la información por ellos aportada, puede ser resumida gráfica-

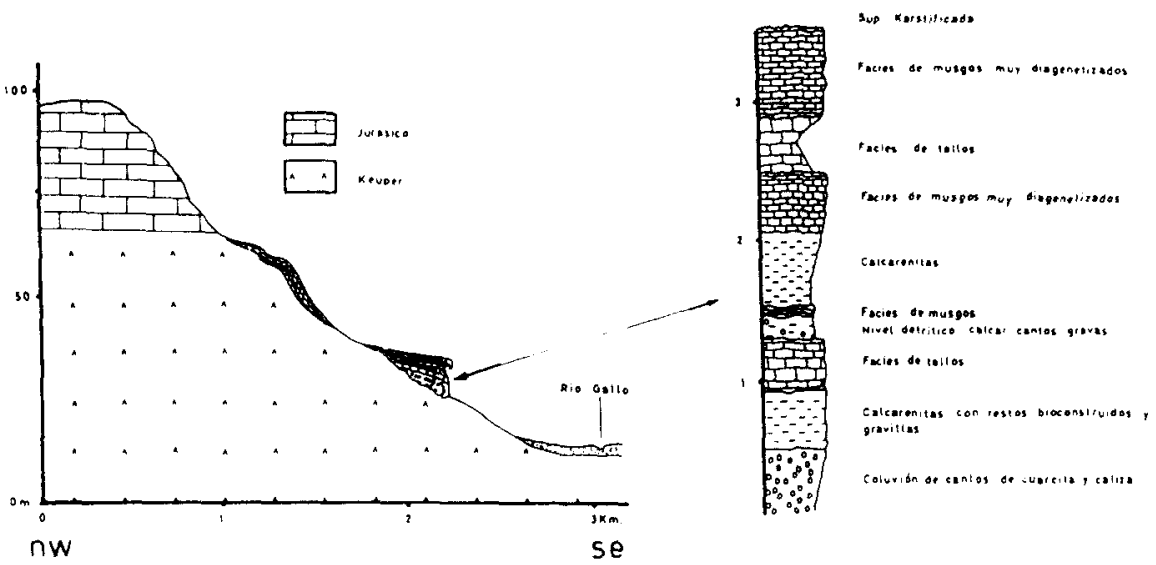

Figura 4. Corte de sintesis del valle del rio Gallo en el sector de Corduente. 
mente en una serie de bloquediagramas, en los que se represente la reconstrucción paleoambiental inferida de los análisis sedimentológicos, petrográficos, etc.
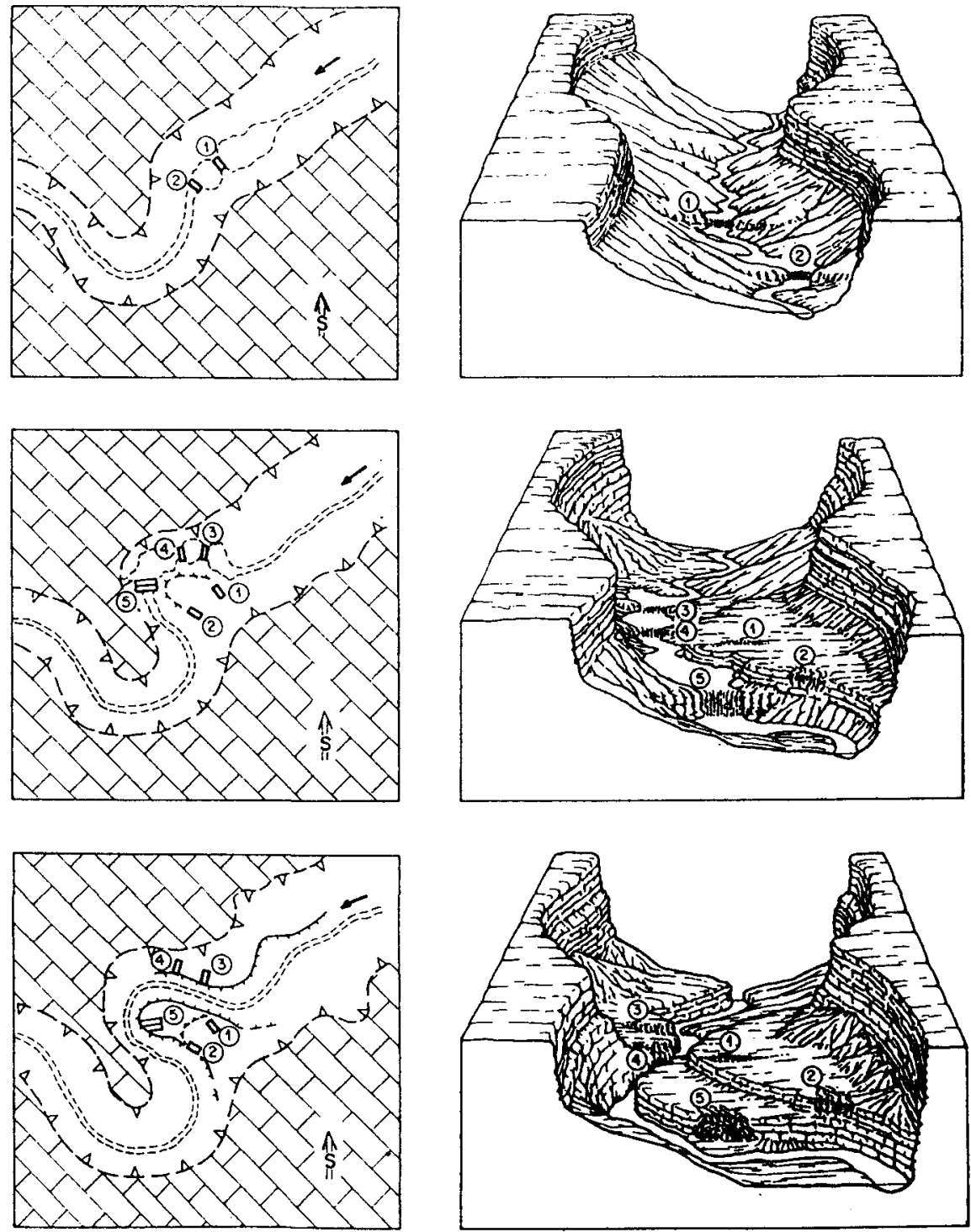

Figura 5. Evolución del valle del Júcar en el sector de Maldonado. 
Es conveniente tener en cuenta los siguientes puntos a la hora de dibujar los bloquediagramas:

1. El número de secuencias evolutivas conviene que sea reducido, de tres a cinco, con la finalidad de no diluir la información evolutiva en múltiples subfases que pudieran introducir confusión interpretativa.

2. Es conveniente centrar la atención en el estado de las laderas, el grado de recubrimiento vegetal y las características hidrográficas (caudal, trazado).

3. Es preciso que los testigos geomorfológicos generados en una etapa aparezcan en la siguiente hasta llegar al momento actual. De esta forma se asegura un nexo de unión a lo largo de la secuencia paisajística.

\section{CONCLUSIONES}

La cartografía geomorfológica es la sintesis espacial de la información obtenida en un trabajo de investigación geomorfológica.

La utilización de la escala adecuada depende de la cartografía base disponible, del volumen de información obtenida y la dimensión del hecho geomorfológico cartografiado.

En ocasiones es necesario completar el uso de los mapas con transectos en los que quede plasmada la información geológica y geomorfológica, e incluso, el levantamiento de bloquediagramas en los que se establezca una secuencia evolutiva.

\section{BIBLIOGRAFIA}

Gonzalez Amuchastegui, M. aJ., y Gonzalez Martin, J.A. (1989): «Geomorfología de las formaciones tobáceas del valle del río Gallo en el área de Molina de Aragón". Cuaternario y Geomorfología. Vol. 3, n. ${ }^{\circ} 1-4, \mathrm{pp}: 63-72$.

Gonzal.ez Amuchastegui, M.aJ. (1993): "Geomorfologia del Alto Tajo en el sector de Molina de Aragón". Tesis doctoral inédita. Universidad Autónoma de Madrid.

FERnÁndez FernánDEZ, A. (1996): “Geomorfología del cañón del río Júcar en la comarca de La Manchuela (Albacete)". Tesis doctoral inédita. Universidad Complutense de Madrid, 1996.

Fernández, A.; Garcia del Cura, M. ․A.; Gonzalez, J.A., y Ordoñez, S. (1996): "Edificios tobáceos actuales en el cauce del rio Júcar (provincia de Albacete)". Geogaceta, n. ${ }^{\circ} 20$ (2). pp. 281-284 